\title{
Fluxon dynamics in long annular Josephson tunnel junctions
}

\author{
Martucciello, N.; Mygind, Jesper; Koshelets, V.P.; Shchukin, A.V.; Filippenko, L.; Monaco, R
}

\section{Published in:}

Physical Review B

Link to article, DOI:

10.1103/PhysRevB.57.5444

Publication date:

1998

Document Version

Publisher's PDF, also known as Version of record

Link back to DTU Orbit

Citation (APA):

Martucciello, N., Mygind, J., Koshelets, V. P., Shchukin, A. V., Filippenko, L., \& Monaco, R. (1998). Fluxon dynamics in long annular Josephson tunnel junctions. Physical Review B, 57(9), 5444-5449.

https://doi.org/10.1103/PhysRevB.57.5444

\section{General rights}

Copyright and moral rights for the publications made accessible in the public portal are retained by the authors and/or other copyright owners and it is a condition of accessing publications that users recognise and abide by the legal requirements associated with these rights.

- Users may download and print one copy of any publication from the public portal for the purpose of private study or research.

- You may not further distribute the material or use it for any profit-making activity or commercial gain

- You may freely distribute the URL identifying the publication in the public portal

If you believe that this document breaches copyright please contact us providing details, and we will remove access to the work immediately and investigate your claim. 


\title{
Fluxon dynamics in long annular Josephson tunnel junctions
}

\author{
N. Martucciello \\ INFM - Dipartimento di Fisica, Università di Salerno, I-84081, Baronissi (SA), Italy \\ J. Mygind \\ Department of Physics, Technical University of Denmark, B309, DK-2800 Lyngby, Denmark \\ V. P. Koshelets, A. V. Shchukin, and L. V. Filippenko \\ Institute of Radio Engineering and Electronics RAS, Mokhovaya 11, Moscow 103907, Russia
}

\author{
Roberto Monaco* \\ Istituto di Cibernetica del C.N.R, I-80072, Arco Felice (NA), Italy \\ and INFM - Dipartimento di Fisica, Università di Salerno, I-84081, Baronissi (SA), Italy
}

(Received 23 September 1997)

\begin{abstract}
Single-fluxon dynamics has been experimentally investigated in high-quality $\mathrm{Nb} / \mathrm{Al}-\mathrm{AlO}_{x} / \mathrm{Nb}$ annular Josephson tunnel junctions having a radius much larger than the Josephson penetration depth. Strong evidence of self-field effects is observed. An external magnetic field in the barrier plane acts on the fluxon as a periodic potential and lowers its average speed. Further, the results of perturbative calculations do not fit the experimental current-voltage profile and, provided the temperature is low enough, this profile systematically shows pronounced deviations from the smooth predicted form. The deviations take the form of fine, hysteretic, almost equally spaced structures. [S0163-1829(98)04406-3]
\end{abstract}

\section{INTRODUCTION}

The soliton (or fluxon) dynamics in annular, i.e., ringshaped Josephson tunnel junctions is an interesting physical phenomenon due to the fluxoid quantization in a superconducting ring and due to the absence of collisions with boundaries. ' This geometry was studied in the mid 1980's (Refs. 2-4) and recently it has gained renewed interest. So far only junctions with a mean radius $\bar{r}$ smaller or comparable to the Josephson penetration depth $\lambda_{J}$ have been considered; for these junctions the Kulik perturbative theory for the Fiske singularities has been successfully extended. ${ }^{5}$ In this paper we investigate the fluxon dynamics in relatively long annular junctions, i.e., when $\bar{r}>\lambda_{J}$, up to $=\bar{r} / \lambda_{J}$ $\approx 10$. In Sec. II we will describe the samples and the experimental setup and we show why the normalized radius, $\rho$, rather than the normalized circumference should be used to properly compare the electrical length of annular junctions with that of linear junctions.

Only the first zero-field step (ZFS1) corresponding to a single fluxon moving around the junction will be investigated, since on higher-order steps the different arrangements of the fluxons inside the barrier and the fluxon collisions make the interpretation of the observed phenomena difficult. In the simplest picture the velocity of a single fluxon, considered as a relativistic particle, is determined by a balance between the driving force on the fluxon and the drag force due to the dissipative losses. In the absence of a magnetic field, the driving force is proportional to the bias current density which is uniform in the junction. The losses are given by the normalized quasiparticle shunt loss coefficient $\alpha$ and the surface losses in the superconducting electrodes described by the surface loss coefficient $\beta$. A relativistic formula for the fluxon motion can be derived ${ }^{1}$ for an infinitely long junction using a perturbational approach. It predicts a smooth current-voltage profile of the ZFS1 branch in annular junctions where, as on an infinite line, the fluxon moves only subjected to cyclic boundary conditions:

$$
\gamma^{-2}=\left(\frac{\pi}{4 \alpha}\right)^{2}\left(u^{-2}-1\right)\left[1+\frac{\beta}{3 \alpha\left(1-u^{2}\right)}\right]^{-2},
$$

where $\gamma$ is the distributed bias current normalized to $2 \pi J_{c} \bar{r} \Delta r . J_{c}$ is the uniform maximum Josephson current density, $\Delta r$ is the ring width, and $u$ is the fluxon speed divided by the Swihart velocity $c_{0}$. Annular Josephson tunnel junctions have been used to test the perturbation model since $1985 .^{3}$ Further, Eq. (1) enables the determination of the temperature dependence of the loss parameters measuring the ZFS1 profile at different temperatures. In Sec. IV we present the changes on the $I-V$ characteristic with temperature and show that the data cannot be fitted by Eq. (1). Provided the temperature is low enough, the ZFS1 profile systematically shows pronounced deviations from the smooth predicted form. These deviations take the form of fine, hysteretic, almost equally spaced structures.

Using a Lagrangian formalism Grønbech-Jensen et al. ${ }^{6}$ have proposed a model for an annular junction in an external magnetic field parallel to the barrier plane. They showed that the field accounts for an additional periodic term in the perturbed sine-Gordon equation which describes the system:

$$
\phi_{x x}-\phi_{t t}-\sin \phi=\alpha \phi_{t}-\beta \phi_{x x t}-\gamma-\eta \Delta \sin x / \rho .
$$

Here $\phi$ is the phase difference of the macroscopic superconducting quantum-mechanical wave functions across the bar- 
rier of the Josephson tunnel junction. The dimensionless angular coordinate $x$ increases by $2 \pi \rho$ upon a round trip and the time has been normalized to the inverse of the maximum junction plasma frequency. $\eta$ is the applied magnetic field normalized to $\Phi_{0} / 2 \pi \mu_{0} \bar{r} d$, and $\Delta$ is the coupling between the external field and the flux density in the junction. Equation (2) was derived in Ref. 7 by requiring that the induction field is continuous at the junction boundary; it was also demonstrated that $\Delta$ depends on the particular electrode configuration, and is always inversely proportional to the square of the normalized radius $\left(\Delta=\rho^{-2}\right.$ for the sample geometry reported in this work). Equation (2) has periodic boundary conditions depending on the number $n$ of fluxons trapped in the annular junction:

$$
\varphi(x+2 \pi \rho)=\varphi(x)+2 \pi n, \quad \varphi_{x}(x+2 \pi \rho)=\varphi_{x}(x),
$$

where $n$ (integer) appears as a topological constant of the system.

It has also been shown ${ }^{8}$ that in the nonrelativistic limit $\left(u^{2} \ll 1\right)$ and disregarding the surface losses, the equation of motion for the soliton regarded as a particle is

$$
\psi_{t t}+\alpha \psi_{t}+\frac{\pi \eta}{4 \rho^{3} \cosh (\pi / 2 \rho)} \sin \psi=\frac{\pi \gamma}{4 \rho},
$$

where $\psi=x_{0} / \rho$ and $x_{0}$ is the instantaneous soliton position. This is the equation of motion for the damped and driven pendulum and can be cast in the more familiar form:

$$
\psi_{t t}+\alpha \psi_{t}+\omega_{0}^{2} \sin \psi=\omega_{0}^{2} \Gamma,
$$

with the natural oscillation frequency $\omega_{0}$ $=\sqrt{\pi \eta / 4 \rho^{3} \cosh (\pi / 2 \rho)}$ depending on the magnetic field $\eta$ and the forcing term $\Gamma=(\gamma / \eta) \rho^{2} \cosh (\pi / 2 \rho)$. We point out that both $\omega_{0}$ and $\Gamma$ also depend on the normalized junction radius $\rho$. In Sec. III we show how the ZFS1 profile depends on the magnetic field and demonstrate that, according to Eq. (3), a magnetic field in the barrier plane reduces the average fluxon speed. The paper is concluded with a short summary.

\section{THE SAMPLES AND THE SETUP}

The high-quality Nb/Al-AlO $O_{x} / \mathrm{Nb}$ Josephson tunnel junction were fabricated with a whole-wafer process in which the junctions are formed in the window of the $\mathrm{SiO}$ insulating layer between the base and the counter electrodes. Details of the fabrication technique have been reported elsewhere. ${ }^{9}$ The ring-shaped junctions, depicted in Fig. 1, had a mean radius $\bar{r} \cong 80 \mu \mathrm{m}$, width $\Delta r=4 \mu \mathrm{m}$ and a geometry very close to the so-called Lyngby geometry ${ }^{3}$ in which both the base and top electrode have a hole concentric to the ring. The idle region surrounding the barrier had a width approximately equal to the junction width on both sides; further, as shown in Fig. 1, the base electrode (dashed line) was slightly larger than the wiring film (full line); this "trick" seems to make fluxon trapping easier. ${ }^{10}$ In fact, by chance, we trapped a single fluxon once every 10 or 20 attempts during which the junction temperature was raised above its critical value by means of a heating resistor placed close to the (unbiased) sample. After this trapping procedure the zero-voltage critical current was at least 20 times smaller than its maximum

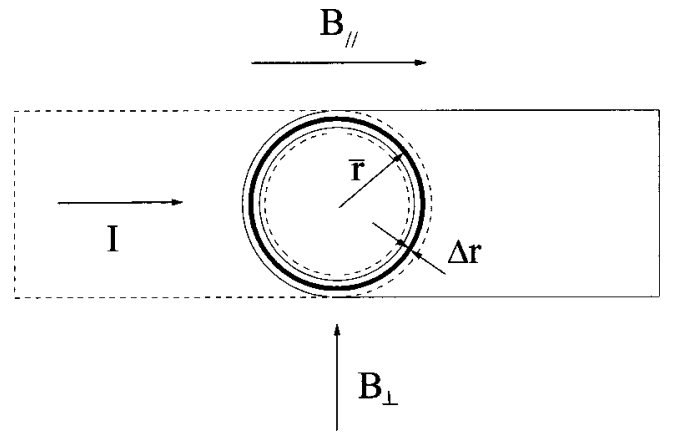

FIG. 1. Schematic of our annular $\mathrm{Nb} / \mathrm{Al}-\mathrm{AlO}_{x} / \mathrm{Nb}$ junctions. The base electrode (dashed line) is slightly larger than the wiring film (full line). The tunnel barrier is shown by the black area.

value. It was possible to trap two fluxons per about 100 attempts. We tried other trapping procedures such as the "field" cooling and the "current" cooling, but we had to reject these methods since they very likely trapped also unwanted Abrikosov vortices in the superconducting films. Abrikosov vortices may act on the fluxon as pinning centers, and thus increase the junction critical current.

Many samples have been measured. For clarity only two will be discussed here. The geometrical and electrical (at 4.2 $\mathrm{K})$ parameters of the two selected annular junctions on different wafers are listed in Table I. They have the same geometry, but differ in the critical current density, i.e., in the normalized radius ( 3.2 and 8.0, for sample $\mathrm{A}$ and $\mathrm{B}$, respectively) and in the maximum plasma frequency. The critical current density has been calculated from the measured quasiparticle current step, $\Delta I_{g}$, at the gap voltage. The magnetic thickness $\Lambda$ was evaluated from the magnetic pattern of small test junctions produced on the same wafer during the same fabrication process. On the same chip a linear overlap junction with the same width, length and idle region was placed in order to measure the junction Swihart velocity $\left(c_{0}=1.4 \times 10^{7} \mathrm{~m} / \mathrm{s}\right)$ which, due to the effect of the idle region, is 1.5 times larger than the bare junction Swihart velocity. The data in Table I show that the junctions A and B are high-quality, long annular Josephson tunnel junctions.

TABLE I. Geometrical and electrical parameters of two selected annular Josephson tunnel junctions at $4.2 \mathrm{~K}$.

\begin{tabular}{ccc}
\hline \hline Sample & A & B \\
\hline Mean radius $\bar{r}(\mu \mathrm{m})$ & 80 & 80 \\
Width $W(\mu \mathrm{m})$ & 4 & 4 \\
Zero field crititical current $I_{o}(\mathrm{~mA})$ & 3.4 & 21.0 \\
Maximum crititical current $I_{\max }(\mathrm{mA})$ & 3.7 & 23.1 \\
Gap quasiparticle current step $\Delta I_{g}(\mathrm{~mA})$ & 6.8 & 48 \\
$I_{\max } / \Delta I_{g}$ & 0.54 & 0.48 \\
Critical current density $J_{c}\left(\mathrm{~A} / \mathrm{cm}^{2}\right)$ & 240 & 1700 \\
Maximum plasma frequency $f_{p}(\mathrm{GHz})$ & 50 & 130 \\
Josephson length $\lambda_{J}(\mu \mathrm{m})$ & 25 & 10 \\
Normalized mean radius $\rho$ & 3.2 & 8.0 \\
Quality factor $V_{m}(\mathrm{mV})$ & 74 & 68 \\
Magnetic thickness $\Lambda(\mathrm{nm})$ & 180 & 180 \\
Junction specific capacitance $c_{s}\left(F / \mathrm{m}^{2}\right)$ & 0.08 & 0.08 \\
ZFS1 asymptotic voltage $(\mu \mathrm{V})$ & 55 & 46 \\
\hline \hline
\end{tabular}


The samples were measured in a very well electrically and magnetically shielded environment. External magnetic fields both parallel and orthogonal to the bias current could be applied simultaneously by means of a solenoid and two Helmoltz coils, respectively. The temperature could be lowered by pumping on the helium bath and the pressure was stabilized with a Cartesian manostat. Above $4.2 \mathrm{~K}$ the temperature could be increased by means of a heater.

\section{Why $\rho$ instead of $l$}

Since we are dealing with junctions long compared to $\lambda_{J}$, we first have to understand how large is the magnetic selffield generated by the bias current, since any external magnetic field acts on the fluxon as a periodic potential. It is well known that the ratio of the measured zero-field critical current $I_{0}$ to the quasiparticle current step at the gap voltage $\Delta I_{g}$, gives an estimate of the self-field generated by the bias current; the smaller this ratio, the larger the self-field. ${ }^{11}$ For short or self-field free junctions this ratio only depends on the temperature and on the electrode materials. Due to strong-coupling effects this ratio should theoretically be equal to 0.71 for $\mathrm{Nb} / \mathrm{Nb}$ junctions at $4.2 \mathrm{~K}$, although in the literature it is usually less than 0.65 .

Samuelsen ${ }^{12}$ has pointed out that the bias current is uniformly distributed around the sample circumferences for electrically small, one-dimensional $(\Delta r \ll \bar{r})$ annular junctions whose electrode width equals the ring diameter. This is because the distribution of the current carried by a superconducting thin film is larger on the film edges and exactly matches the junction current per unit length; in fact, they both vary as $1 / \sqrt{\vec{r}^{2}-y^{2}}$ in which $y$ is the transverse electrode coordinate. Due to this coincidence annular junctions with small normalized circumference have the ratio of the zero-field critical current to the gap quasiparticle current step close to that of a small junction. Martucciello et al. ${ }^{7}$ found that the largest critical current $I_{\max }$ of long annular junctions can be obtained by applying a magnetic field in the direction perpendicular to that of the bias current in order to partially compensate the self-field. From Table I we see that the $I_{\max }$ is about $10 \%$ larger than $I_{o}$ for both samples. However, the ratio $I_{\max } / \Delta I_{g}$, although large for both samples, decreases with the normalized circumference of the ring. This indicates that the self-field has a complex spatial dependence that cannot be fully compensated by the application of an uniform external field as in the case of linear junctions. ${ }^{11} \mathrm{We}$ conclude that long annular junctions feel the presence of a nonuniform magnetic field proportional to the bias current in the junction itself; the longer the junction, the larger is the effect, just as in linear junctions. This is also supported by the numerically computed magnetic patterns for annular junctions having different normalized radii. ${ }^{7}$ The normalized radius $\rho$ (and not $l$ ) has to be larger than unity to get a noticeable discrepancy from the small junction behavior. Further, it has also been analytically shown ${ }^{13}$ that the critical field, $\eta_{c}$, of the annular junctions follows a quadratic dependence on $\rho$ : $\eta_{c}(\rho)=\eta_{c}(0)+\rho^{2}$. Another manifestation of the self-field is found by looking at the ZFS1 asymptotic voltages of the two samples. For sample A $V_{\mathrm{ZFS} 1}$ is very close to the value expected when assuming that the Swihart velocity is that measured on the overlap junction $\left(c_{0}=1.4 \times 10^{7} \mathrm{~m} / \mathrm{s}\right)$. For the

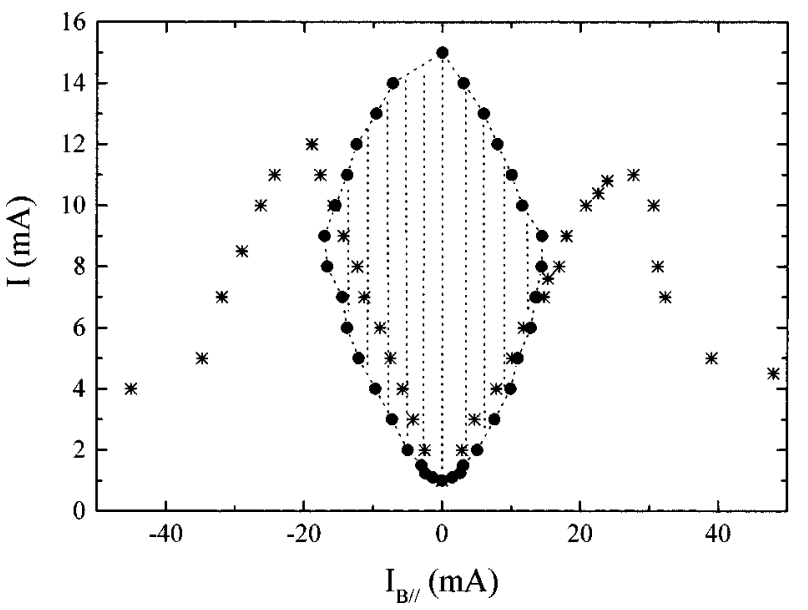

FIG. 2. Magnetic pattern of the critical current (stars) for sample B with one trapped fluxon in a current-parallel magnetic field $B_{\|}$ proportional to the current $I_{B_{\|}}$flowing in the coil. The hatched area delimited by the dots shows the range of existence of the traveling fluxon mode in the $I-B_{\|}$plane.

longer samples this voltage is considerably lower suggesting that the soliton moves in a periodic potential, as will be shown in the next section. Although qualitative considerations bear strong evidence of the self-field effect, a quantitative analytical calculation of the current and field distribution inside a long annular junction has not been made.

In order to minimize the effect of the magnetic self-field, a proper external field $B_{\perp}$ in the direction perpendicular to the bias current and with an amplitude proportional to the bias current itself was applied in all the measurements presented in this work.

\section{MAGNETIC MEASUREMENTS}

\section{A. Magnetic patterns}

Figure 2 shows for sample B with one trapped fluxon at $T=1.8 \mathrm{~K}$, the dependence of both the critical current $I_{o}$ (stars) and the amplitude of the ZFS1 (dots) as a function of an external magnetic field $B_{\|}$parallel to the bias current ( $B_{\|}$is proportional to the current $I_{B_{\|}}$flowing in the coil). We observe that although small (less than $5 \%$ of $I_{\max }$ ), the zerofield critical current is not zero as theoretically expected for an ideal tunnel barrier where an infinitesimal bias current drives the fluxon away leaving the junction in a finite voltage state. This indicates that the fluxon gets pinned in a small potential due to unavoidable imperfections in the real barriers. When the $B_{\|}$field is increased, then the potential well grows deeper and a larger driving force, i.e., bias current, is needed to start the fluxon motion; the $I_{o}$ vs $B_{\|}$dependence follows, at least qualitatively, the first-order Bessel function behavior expected for small annular junctions. ${ }^{13}$ As far as the ZFS1 is concerned, we found that it becomes unstable if an external field is applied, since the fluxon gets stuck in the potential well. Figure 2 clearly shows the stability range of the fluxon in the $I-B \|$ plane (hatched area). The upper curve was obtained by fixing the $B$ value and increasing the current until the junction switches to higher voltages and, vice versa, the lower curve was obtained by setting the $I$ value and increasing the parallel field until the fluxon gets pinned in the 

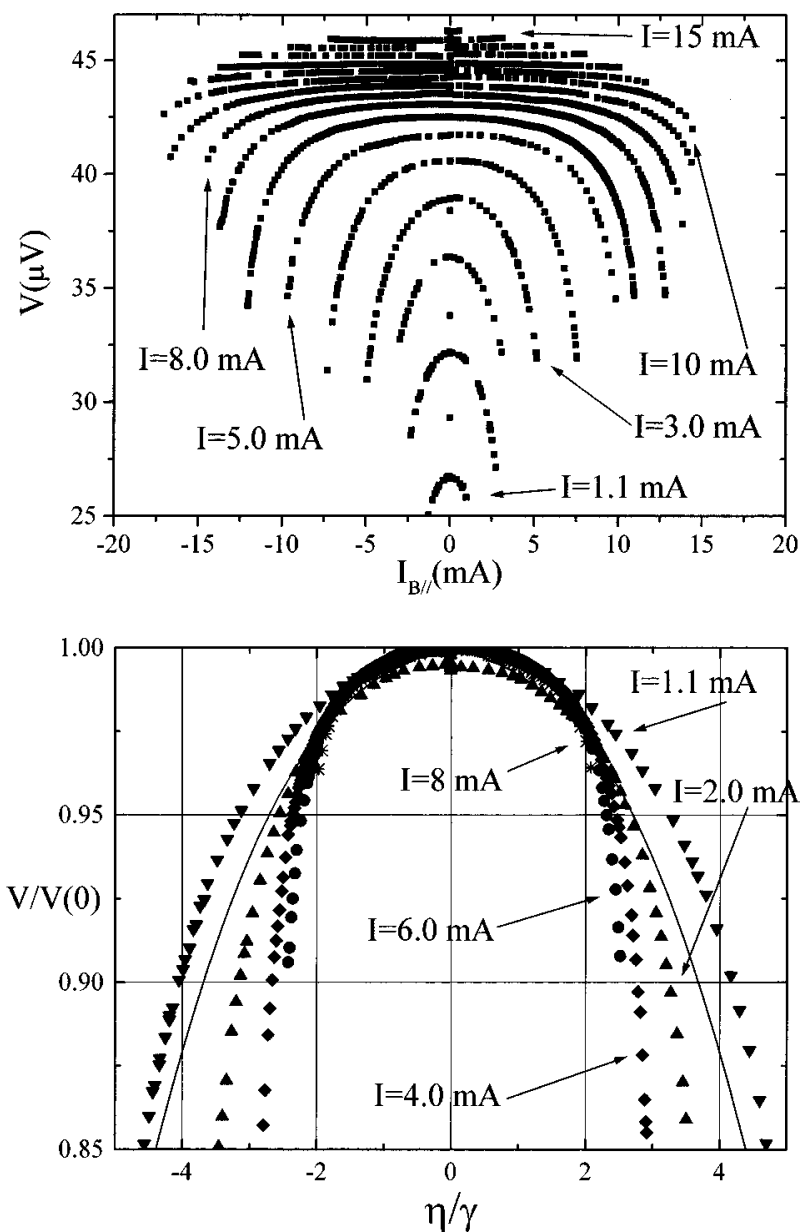

(b)

FIG. 3. (a) Magnetic tuning of the single fluxon voltage at different bias points along the ZFS1 for sample B at $T=4.2 \mathrm{~K}$. (b) Low-bias data of (a) in normalized units; for each bias current $\mathrm{V}(0)$ is the step voltage in zero magnetic field. The full line is the analytic expression (4).

potential well and the junction voltage switches to zero. Similar data have been obtained for negative bias current.

\section{B. Magnetic tuning}

The ZFS1 voltage can be tuned by the external field. Figure 3(a) shows for different bias currents along the ZFS1 the voltage shift due to a magnetic field applied in the direction of the bias current ( $B_{\|}$is proportional to the current $I_{B_{\|}}$flowing in the coil). A dc voltage shift as small as $200 \mathrm{nV}$ was easily observed. We see that the $B_{\|}$field causes a negative tuning (i.e., a reduction of the dc voltage, and hence of the average fluxon speed), except on the very top of the step where the tuning is slightly positive. Further, the tuning is more effective at lower bias, although the magnetic field can be changed in a larger range as we move to a higher bias current. A relative tuning as large as $20 \%$ has been measured at the middle of the step. The tuning is rather symmetric with respect to field reversal, and similar data have been obtained also for the negative step corresponding to the single fluxon moving in the opposite direction. In Fig. 3(b) we have plotted the low-bias data of Fig. 3(a) normalizing the voltages to the corresponding zero-field voltage and dividing the field values by a quantity proportional to the bias current. In this way all the data fall close to each other; indeed the data progressively shrink as we move to a higher bias current. This agrees with the theory where in the nonrelativistic limit a fluxon moving on an annular junction in a barrier parallel magnetic field is analog to a driven and damped pendulum, the average fluxon speed being related to the pendulum rotation frequency. Unfortunately an analytic expression for the dependence of the rotation frequency on the amplitude of the gravitational field only exists in the case of quadratic losses, i.e., with $\alpha \phi_{t}$ replaced with $\alpha \phi_{t}^{2}$ in Eq. (3). ${ }^{14}$ Simple algebraic manipulations show that

$$
\frac{u(\chi)}{u_{0}}=\frac{\sqrt{1+\chi}}{(2 / \pi) K[2 \chi /(1+\chi)]},
$$

where $K(m)$ is the complete elliptic integral and the argument $\chi=2 \alpha \eta / \gamma \rho^{2} \cosh (\pi / 2 \rho)$ is proportional to the ratio $\eta / \gamma$, confirming the universal behavior found in the experiments. Equation (4) is plotted as a full line in Fig. 3(b). The agreement with the experimental data is quite satisfactory.

We point out that the viscous losses play an important role for the tuning. In the classical limit and in absence of losses the average fluxon speed would be independent of the external field since it creates a potential which only modulates its speed around its zero-field value without affecting the average. However, in the presence of viscous losses the soliton in a periodic potential in the average dissipates more energy resulting in a decrease of the average speed. Indeed, we have experimentally found that the tuning rate is smaller at lower temperatures where also the losses are smaller. The results shown in Fig. 3 are qualitatively similar to those expected in long linear junctions ${ }^{15}$ for which the magnetic field is known to reduce the fluxon energy.

\section{TEMPERATURE MEASUREMENTS}

\section{A. Loss determination}

Figure 4(a) shows for sample A at different temperatures the current-voltage characteristic of the first zero-field singularity, that is, when the junction has one fluxon trapped in the barrier and no external field is applied. The step spans a very large voltage range and has a return current always lower than $0.1 \mathrm{~mA}$. The shape of the step provides information about the losses experienced by the soliton. According to Eq. (1) $\alpha$ and $\beta$ can be found by plotting the inverse of the squared current as a function of the inverse of the squared voltage. In the absence of $\beta$ losses this plot should be a straight line; deviations from a straight line are expected at high voltages $\left(v^{-2} \rightarrow 1\right)$, the shunt loss parameter $\alpha$ being strictly related to the curve slope at low voltages $\left(v^{-2}\right.$ $\rightarrow \infty$ ). Figure 4(b) shows the same step profiles plotted as $\gamma^{-2}$ vs $v^{-2}$. Unfortunately, a comparison between the experimental data and the perturbation theory is impossible since they have opposite concavity; in fact, $d^{2} \gamma^{-2} / d\left(v^{-2}\right)^{2}$ is always negative for the experimental data, while it should be positive for nonzero $\beta$ in Eq. (1). Further, the analysis of the slope $d \gamma^{-2} / d v^{-2}$ in the low voltage limit yields unreasonably large $\alpha$ values. This indicates that the infinite length perturbation theory does not apply to long annular junctions. Similar results have been found for sample B. A good fit between Eq. (1) and experimental data on $\rho \approx 2, \mathrm{Nb} / \mathrm{Pb}$ an- 
(a)
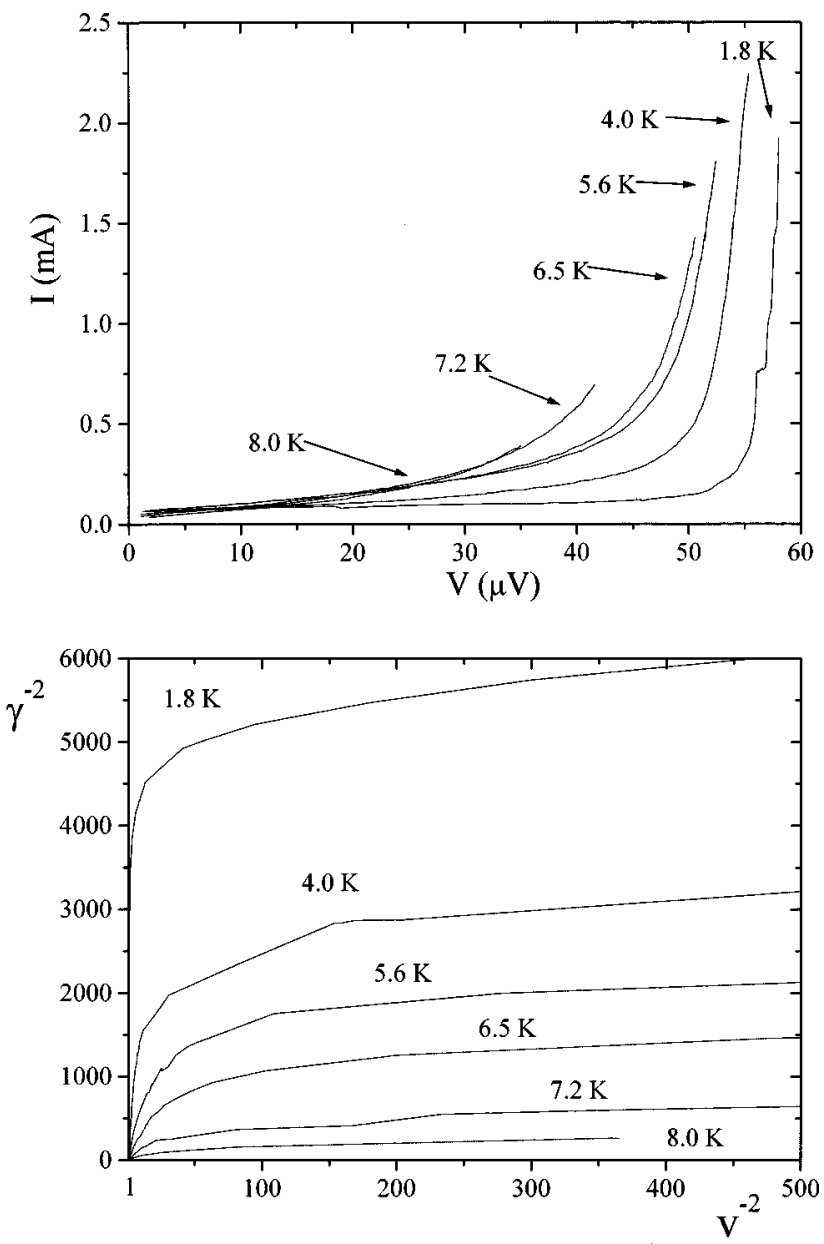

(b)

FIG. 4. (a) ZFS1 profile for sample A at different temperatures; (b) the same data of (a) plotted as $\gamma^{-2}$ versus $v^{-2} \cdot \gamma$ is the distributed bias current $I$ normalized to $I_{0}(T)$ and $v$ is the junction voltage $V$ divided by the temperature-dependent asymptotic voltage.

nular junction has been reported in Ref. 4, but only in a very small voltage range close to the asymptotic voltage (1 $\leqslant v^{-2} \leqslant 1.5$ ) where deviations from the perturbation theory are expected to occur. Also our data show a very large slope in this range. We point out that this is an attempt to apply the perturbation theory to high-quality $\mathrm{Nb} / \mathrm{Al}-\mathrm{AlO}_{x} / \mathrm{Nb}$ junctions and over a very large voltage range.

\section{B. Fine structures at low temperatures}

In Fig. 4(a) the step profile becomes increasingly steeper as the temperature decreases and eventually fine structures appear in the form of hysteretic, tiny steps. Figure 5 shows the low-temperature ZFS1 for sample B and the inset shows that the voltages at which the fine structures occur are almost equally spaced, with the voltage spacing of about $0.4 \mu \mathrm{V}$. A differential resistance $d V / d I$ as low as $40 \mu \Omega$ has been measured on the top of the step. Further, fine structures have been observed also on the fluxon-antifluxon step in the case of no trapped fluxons; In this case, in spite of the fluxonantifluxon collisions, we found a similar step profile provided that a factor of 2 in the voltage scale was considered due to the presence of two traveling solitons.

No external magnetic field was applied to the annular junctions in order to observe the fine structures which there-

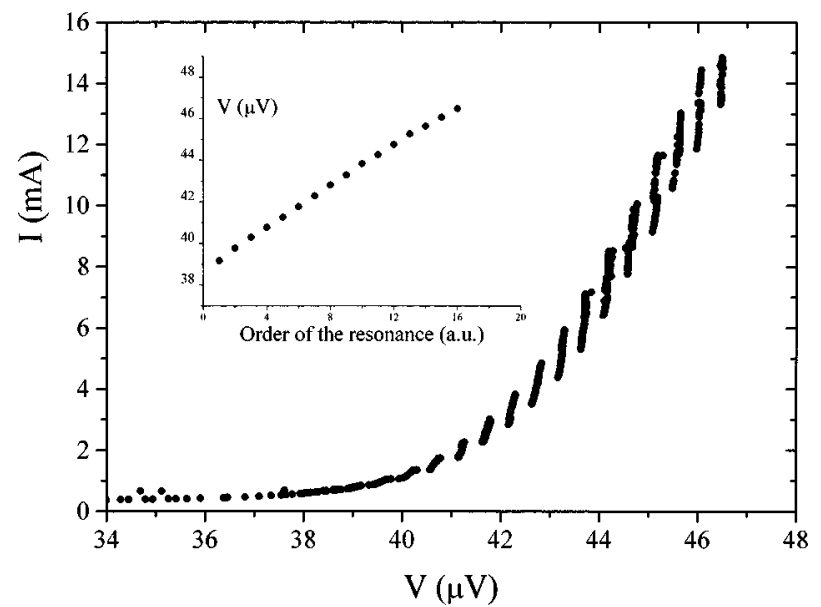

FIG. 5. Example of fine structures on the profile of the zero-field singularity for sample B measured at $T=1.8 \mathrm{~K}$. The inset demonstrates that the fine structures are almost equally spaced.

fore are different from those found numerically by Grønbech et $a .^{8}$ and recently experimentally reported by Ustinov ${ }^{10}$ on the ZFS1 profile of annular junctions in the presence of an externally applied field. In this case, a resonance may occur between the traveling fluxon and the plasma wave emitted by the fluxon itself while it is accelerated in the periodic potential. In our case, the application of an external field did not induce the appearance of new structures, but it only caused a tuning of the zero-field resonances as described in the previous section. Therefore, we do not believe that the fine structures in long annular junctions are due to the self-field, even though they are more pronounced in longer junctions.

The fine structures have often been reported since 1981 on the zero-field singularities of a long linear junction ${ }^{16-18}$ and several models have been proposed to explain them. Chang et al. ${ }^{16}$ related the fine structures to the interaction of $p$ fluxon oscillations with the $q$ th fractional cavity mode, assuming the response of the Josephson resonator to be linear. In this case, the resonant frequencies should be a rational fraction of the characteristic frequency $f_{c}=c_{o} /(2 \pi r) \approx 30$ $\mathrm{GHz}$, but unreasonable numbers for $p$ and $q$ where necessary. Later, taking into account the nonlinear nature of a Josephson cavity, the fine structure was ascribed to the interaction of the traveling fluxon with small amplitude plasma oscillations with the frequency $f_{p}=1 / 2 \pi \sqrt{2 e I_{c} / \hbar C}$ (where $C$ is the junction capacitance and $I_{c}$ is its critical current). In this case, the resonant frequencies should occur at $f_{n}$ $=f_{p} / n \cdot{ }^{17}$ In a recent paper Barbara et al. ${ }^{18}$ described the development of the fine structures at low temperatures as due to the interaction between the fluxon and the plasma waves generated by the fluxon itself during the acceleration experienced at reflection on the junction edges. Unfortunately none of the previous explanations seem to be able to fit the voltage position of the fine structures we observed. Even an attempt assuming the presence of one or two (diametrically opposed) inhomogeneities in the barrier failed.

\section{SUMMARY}

We have studied the single soliton dynamics on long $\mathrm{Nb} / \mathrm{Al}-\mathrm{AlO}_{x} / \mathrm{Nb}$ annular Josephson tunnel junctions (Fig. 1) 
in the presence of an external field applied in the plane of the barrier or at different temperatures. We found that in order to partially compensate the effect of the self-induced magnetic field an external field $B_{\perp}$ with an amplitude proportional to the bias current was needed in the direction perpendicular to the current flow in the electrodes. As a result we obtained symmetric dependences of the step amplitudes (Fig. 2) and of the voltage tuning [Fig. 3(a)] on the amplitude of an external field $B \|$ parallel to the bias current. Due to the presence of viscous losses, the average fluxon speed is reduced when it moves in the periodic potential generated by the uniform external field according to Eq. (2). We have fitted our data assuming that the soliton behaves as a classical particle in the presence of ohmic losses, Indeed fitting is not possible at large bias currents where the effect of the surface loss cannot be neglected.

Further, studying the fluxon dynamics at different tem- peratures, we found that our $I-V$ characteristics cannot be explained by the perturbation theory Eq. (1) (Fig. 4) and, even more surprisingly, fine structures develop on the $I-V$ profile at low temperatures (Fig. 5).

\section{ACKNOWLEDGMENTS}

The authors wish to thank N.F. Pedersen and M.R. Samuelsen for stimulating discussions and M.H. Manscher for valuable technical assistance. This work was partially supported by the Consiglio Nazionale delle Ricerche under the Progetto Finalizzato Superconductive and Cryogenic Technologies, by the Danish Natural Science Foundation, by the Russia Program for Basic Research, the Russian State Scientific Program, Superconductivity, and NATO Grant HTECH CRG No. 960088.
*Electronic address: roberto@vaxsa.csied.unisa.it

${ }^{1}$ D. W. McLaughlin and A. C. Scott, Phys. Rev. A 18, 1652 (1978).

${ }^{2}$ B. Dueholm, A. Davidson, C. C. Tsuei, M. J. Brady, K. H. Brown, A. C. Callegari, M. M. Chen, J. H. Greiner, H. C. Jones, K. K. Kim, A. W. Kleinsasser, H. A. Notarys, G. Proto, R. H. Wang, and T. Yogi, in Proceedings of LT-17, edited by U. Eckerm, A. Schmid, W. Weber, and H. Wühl (Elsevier Science, Amsterdam, 1984).

${ }^{3}$ A. Davidson, B. Dueholm, B. Kryger, and N. F. Pedersen, Phys. Rev. Lett. 55, 2059 (1985).

${ }^{4}$ A. Davidson, B. Dueholm, and N. F. Pedersen, J. Appl. Phys. 60, 1447 (1986).

${ }^{5}$ N. Martucciello, C. Soriano, and R. Monaco, Phys. Rev. B 55, 15 157 (1997).

${ }^{6}$ N. Grønbech-Jensen, P. S. Lomdahl, and M. R. Samuelsen, Phys. Lett. A 154, 14 (1991).

${ }^{7}$ N. Martucciello and R. Monaco, Phys. Rev. B 53, 3471 (1996).
${ }^{8}$ N. Grønbech-Jensen, P. S. Lomdahl, and M. R. Samuelsen, Phys. Rev. B 43, 12799 (1991).

${ }^{9}$ V. P. Koshelets, A. V. Shchukin, I. L. Lapytskaya, and J. Mygind, Phys. Rev. B 51, 6536 (1995).

${ }^{10}$ A.V. Ustinov, JETP Lett. 64, 191 (1996).

${ }^{11}$ A. Barone and G. Paternò, Physics and Applications of the Josephson Effect (Wiley, New York, 1982).

${ }^{12}$ M. R. Samuelsen (private communication).

${ }^{13}$ N. Martucciello and R. Monaco, Phys. Rev. B 54, 9050 (1996).

${ }^{14}$ N.F. Pedersen and K. Saermark, Physica (Amsterdam) 69, 572 (1973).

${ }^{15}$ O.A. Levring, N.F. Pedersen, and M.R. Samuelsen, J. Appl. Phys. 54, 987 (1983).

${ }^{16}$ J. J. Chang, J. T. Chen, M. R. Scheuermann, and D. J. Scalapino, Phys. Rev. B 31, 1658 (1985).

${ }^{17}$ M. R. Scheuermann, C. C. Chi, N. F. Pedersen, J. J. Chang, and J. T. Chen, Appl. Phys. Lett. 48, 189 (1986).

${ }^{18}$ P. Barbara, R. Monaco, and A.V. Ustinov, J. Appl. Phys. 79, 326 (1996). 